\title{
Sistemas de polinização e de reprodução de três espécies de Jatropha (Euphorbiaceae) na Caatinga, semi-árido do Brasil ${ }^{1}$
}

\author{
EDINALDO LUZ DAS NEVES 2 , ISABEL CRISTINA MACHADO ${ }^{3} \mathrm{e}$ \\ BLANDINA FELIPE VIANA ${ }^{4,5}$
}

(recebido: 06 de maio de 2010; aceito: 22 de novembro de 2011

\begin{abstract}
Pollination and reproductive systems of three species of Jatropha (Euphorbiaceae) in the semi-arid Caatinga region of Brazil). The floral biology, nectar production behavior, floral visitors, and reproductive systems of Jatropha ribifolia (Pohl) Baill. (Euphorbiaceae) is presented and compared of those of J. mollissima (Pohl) Baill. and J. mutabilis (Pohl) Baill. The study was undertaken in an area of hyperxerophilous caatinga at the Estação Biológica de Canudos, Bahia State, Brazil, from May 2005 to June 2007. The flowers of the three species are disposed in a protogynic dichasium. In J. ribifolia both staminate and pistilate flowers last for about 48 hours and flower opening is diurnal, while in J. mollissima and J. mutabilis the staminate flowers last from 12 to 15 hours and the pistilate flowers from 36 to 48 hours, with flower opening occurring at sunset. Nectar production, pollen viability, and stigma receptivity initiate soon after full opening of both floral types, and sexual activity overlaps until senescence. Nectar production varied throughout the day, with higher production by the pistilate flowers; pollen viability and stigma receptivity during the lifetime of the flower occurred in all three species. Significant differences occurred between the reproductive system treatments of J. mollissima $(\mathrm{KW}=59.796)$, J. mutabilis $(\mathrm{KW}=59.058)$, or $J$. ribifolia $(\mathrm{KW}=63.660)$. The three species produced fruits by experimental geitonogamy as well as experimental xenogamy, while only $J$. ribifolia produced fruits by spontaneous geitonogamy. The bees Apis mellifera, Xylocopa frontalis and X. grisescens and the hummingbirds Chlorostilbon lucidus and Anopetia gounellei are potential pollinators for J. mollissima and $J$. mutabilis and A. mellifera and X. grisescens are potential pollinators for J. ribifolia. The diversity of anthophiles on flowers of the three species allows pollen flow and fruit set. On the other hand asynchronous flowering and differences of flower morphology and anthesis help maintain the reproductive isolation and prevent the loss of interspecific pollen due to sharing of pollinators.
\end{abstract}

Key words - bees, floral biology, melittophily, ornithophyly, reproductive system

RESUMO - (Sistemas de polinização e de reprodução de três espécies de Jatropha (Euphorbiaceae) na Caatinga, semi-árido do Brasil). São apresentados a biologia floral, a dinâmica de produção de néctar, visitantes florais e sistemas reprodutivos de Jatropha ribifolia (Pohl) Baill. (Euphorbiaceae) e uma comparação dos dados obtidos para J. mollissima (Pohl) Baill. e $J$. mutabilis (Pohl) Baill. O estudo foi desenvolvido em uma área de caatinga hiperxerófila, na Estação Biológica de Canudos, Bahia, Brasil, de maio de 2005 a junho de 2007. As flores das três espécies estão organizadas em dicásios protogínicos. Em J. ribifolia as flores estaminadas e pistiladas duram cerca de 48 horas e a abertura é diurna, enquanto em J. mollissima e $J$. mutabilis as estaminadas duram entre 12 e 15 horas e as pistiladas entre 36 e 48 horas e a abertura é crepuscular. A produção de néctar, a viabilidade polínica e a receptividade estigmática iniciaram-se logo após a abertura total das flores e se sobrepuseram até sua senescência. A produção de néctar variou ao longo do dia e as flores pistiladas produziram maiores volumes que as estaminadas. A viabilidade polínica e a receptividade estigmática foram observadas nas três espécies durante toda a vida da flor. Houve diferença significativa entre os tratamentos dos sistemas reprodutivos para J. mollissima $(\mathrm{KW}=59,796)$, J. mutabilis $(\mathrm{KW}=59,058)$ e $J$. ribifolia $(\mathrm{KW}=63,660)$. As três espécies produziram frutos por geitonogamia manual e xenogamia manual e apenas J. ribifolia produziu frutos por geitonogamia espontânea. As abelhas Apis mellifera, Xylocopa frontalis e X. grisescens e os beija-flores Chlorostilbon lucidus e Anopetia gounellei são os polinizadores potenciais de J. mollissima e J. mutabilis. Já para J. ribifolia, os potenciais polinizadores são A. mellifera e X. grisescens. A utilização de uma gama variada de vetores de pólen permite o fluxo polínico e a formação de frutos nas três espécies. Por sua vez, o sequenciamento no período da floração e diferenças nas dimensões das flores e nos horários da antese ajudam a manter o isolamento reprodutivo das três espécies e evitam a perda de pólen interespecífico, que poderia ser alta devido à partilha de alguns dos polinizadores.

Palavras-chave - abelhas, biologia floral, melitofilia, ornitofilia, sistema reprodutivo

1. Parte da tese de doutorado do primeiro autor, Programa de Pós-Graduação em Botânica da Universidade Estadual de Feira de Santana (UEFS), BA, Brasil.

2. Universidade Estadual de Feira de Santana (UEFS), Departamento de Ciências Biológicas, Rodovia BR116 km 03, Campus Universitário, 44031-460 Feira de Santana, BA, Brasil.
3. Universidade Federal de Pernambuco (UFPE), Departamento de Botânica, Rua Prof. Nelson Chaves, s/n, 50372-970 Recife, PE, Brasil.

4. Universidade Federal da Bahia (UFBA), Instituto de Biologia, Departamento de Zoologia, Grupo de Ecologia de Comunidades de Visitantes Florais. Rua Barão de Geremoabo, s/n, Campus Universitário de Ondina, 40170-110 Salvador, BA, Brasil.

5. Autor para correspondência: blande.viana@gmail.com 


\section{Introdução}

Plantas com flores apresentam diferentes sistemas sexuais e reprodutivos, e esses são variáveis e flexíveis (Proctor et al. 1996). A compreensão desses sistemas ajuda a esclarecer importantes aspectos da interação flor-visitante, do padrão de fluxo gênico das populações, assim como das relações filogenéticas entre espécies vegetais (Richards 1997). Nos ambientes tropicais predominam espécies monoclinas, mas com estratégias florais que dificultam a autogamia e otimizam a xenogamia, normalmente associadas à morfologia floral (Proctor et al. 1996). As características florais podem facilitar ou dificultar a ação dos visitantes seja pela associação entre o tamanho corporal dos animais e as dimensões florais ou pela localização dos recursos na flor e quando essas características convergem, pode resultar na polinização (Frankie et al. 1983).

A família Euphorbiaceae é considerada a terceira família com maior riqueza de espécies na caatinga (Giulietti et al. 2006) e possui representantes com grande potencial agrícola na produção de biocombustível, como Jatropha curcas L. (pinhão manso) e Ricinus communis L. (mamona). Entre os representantes dessa família, a monoicia é um sistema sexual relativamente comum, especialmente nos gêneros Euphorbia, Cnidosculus, Croton e Jatropha (Bullock 1985). Estudos que abordam aspectos da polinização e da biologia reprodutiva de espécies de Jatropha são raros em todo o mundo (e.g. Dehgan \& Webster 1979, Reddi \& Reddi 1983, Ormond et al. 1984, Epila-Otara 1993, Raju \& Ezradanam 2002, Bhattacharya et al. 2005). Para as áreas de caatinga, Santos et al. (2005) estudaram a fenologia floral, biologia floral e o sistema reprodutivo de Jatropha mollissima (Pohl) Baill. e J. mutabilis (Pohl) Baill. nos municípios pernambucanos de Buíque e Arcoverde. $\mathrm{Na}$ Ecorregião do Raso da Catarina, registram-se apenas o trabalho de Neves et al. (2010), que estudaram os comportamentos fenológicos, a capacidade de armazenamento de água e o padrão de distribuição espacial de Jatropha mollissima (Pohl) Baill., J. mutabilis (Pohl) Baill e J. ribifolia (Pohl) Baill. e Neves \& Viana (2011) que testaram experimentalmente a eficiência da Apis mellifera Linnaeus na polinização de $J$. mollissima e $J$. mutabilis em apenas uma visita. Nesse sentido, o presente trabalho apresenta, pela primeira vez, a biologia da polinização de J. ribifolia, contribui com novos dados para $J$. mollissima e $J$. mutabilis e compara as estratégias de polinização das três espécies.

\section{Material e métodos}

Área de estudo - O trabalho de campo foi desenvolvido na Ecorregião do Raso da Catarina (sensu Velloso et al. 2002), área prioritária para a conservação da flora e fauna de invertebrados da caatinga, de acordo com o relatório do Ministério do Meio Ambiente (2002). O clima da região é tropical semi-árido, com temperaturas médias anuais variando entre $27^{\circ} \mathrm{C}$ e $29^{\circ} \mathrm{C}$ e médias de precipitação inferiores a $800 \mathrm{~mm}$ (Inmet - IV Distrito). As coletas de dados foram realizadas no período de maio de 2005 a junho de 2007 na Estação Biológica de Canudos (9॰56’34” S, 3859'17” W), Canudos-BA, pertencente à Fundação Biodiversitas e com área total de 1500 ha. O local possui formações de arenito que produzem canyons e é colonizada por vegetação de caatinga hiperxerófila arbustiva-arbórea.

Espécies estudadas - Jatropha mollissima, J. mutabilis e $J$. ribifolia são arbustos co-ocorrentes na Ecorregião do Raso da Catarina, apresentam comportamento fenológico contínuo e variável quanto à frequência, duração e regularidade dos episódios, com altos índices de sobreposição das fenofases e ausência dos dois tipos de flores (estaminadas e pistiladas) nos meses mais frios e úmidos e de flores pistiladas em momentos isolados do ciclo fenológico (Neves et al. 2010).

Biologia floral - Foram utilizadas 20 flores de cada espécie distribuídas em cinco indivíduos (quatro flores por indivíduo) e registraram-se informações sobre a forma, diâmetro e profundidade da corola, cor, estruturas reprodutivas e sua disposição na flor. Para a medida do comprimento da inflorescência foram selecionadas 20 inflorescência de cada espécie, sendo uma de cada indivíduo. Voucher das espécies foram depositados no Herbário da Universidade Estadual de Feira de Santana (J. mutabilis E.L. Neves 01, J. ribifolia E.L. Neves 02, J. molissima E.L. Neves 03). Durante os períodos de pico de floração (segundo Neves et al. 2010) em 20 indivíduos de cada espécie, previamente marcados, acompanhou-se a duração de uma inflorescência por indivíduo, contou-se o número de flores estaminadas e pistiladas por inflorescência $(n=30)$ e o número de frutos produzidos por inflorescência $(n=30)$. A duração da antese e a longevidade dos dois tipos de flores foram verificadas em 20 flores de cada tipo em 10 indivíduos (duas flores por indivíduo) diferentes para cada espécie.

Utilizando-se 10 botões de cada espécie, em fase de pré-antese e coletados de indivíduos diferentes (um botão de cada indivíduo), contou-se o número de grãos de pólen por antera em microscopia óptica. De cada botão foram retiradas duas anteras e a contagem foi realizada para cada uma delas separadamente. Para testar a viabilidade polínica, utilizou-se 10 flores de cada espécie (uma por indivíduo) coletadas a cada 60 minutos, a partir do início da antese, e armazenadas em microtubos individuais contendo solução de vermelho neutro $1 \%$, de acordo com os procedimentos propostos por Kearns \& Inouye (1993). Em laboratório, foram confeccionadas lâminas semi permanentes para realizar a quantificação 
do percentual de grãos corados sob microscopia óptica. A receptividade do estigma foi detectada utilizando-se peróxido de hidrogênio, de acordo com Dafni et al. (2005). O teste foi realizado em 20 flores de 10 indivíduos diferentes (duas flores por indivíduo), desde o início da abertura da flor até a queda de todas as pétalas nas flores pistiladas ou abscisão das flores estaminadas.

O volume do néctar foi medido em 10 flores estaminadas e 10 pistiladas, por cada espécie (uma flor por indivíduo), utilizando-se microcapilares de 10 e $25 \mu \mathrm{L}$ para J. mollissima e J. mutabilis e de $5 \mu \mathrm{L}$ para J. ribifolia. Para a medida do volume total de néctar produzido, botões foram ensacados com voal para evitar a presença de visitantes. O néctar foi medido 36 horas após o início da antese nas flores pistiladas e após 14 horas nas flores estaminadas. Para avaliar o padrão diário de produção de néctar, botões foram ensacados com voal para evitar a presença de visitantes e o néctar foi medido a cada três horas. Esse procedimento não foi feito para $J$. ribifolia porque as flores eram danificadas logo na primeira amostragem. Para avaliar a quantidade de néctar disponível aos visitantes (nectar standing crop), realizou-se a medida de seis em seis horas em flores funcionais abertas aos visitantes. A concentração de solutos foi medida utilizandose refratômetro de bolso (Atago ${ }^{\circledR} 0 \%$ a $32 \%$ ), sendo o total de açúcar (em mg) mensurado utilizando-se a tabela proposta por Galetto \& Bernadello (2005).

Sistemas reprodutivos - Para avaliar os sistemas reprodutivos, realizaram-se os seguintes experimentos que foram acompanhados até a maturação dos frutos ou abscisão das flores: (1) geitonogamia espontânea, ensacando-se 20 inflorescências contendo botões de flores pistiladas e estaminadas e deixando-os sem manipulação, (2) apomixia, 20 botões de flores pistiladas foram ensacados no dia anterior à antese e deixados sem manipulação, (3) geitonogamia manual, 20 botões de flores pistiladas foram ensacados no dia anterior à antese e, após a abertura da flor, realizou-se a polinização manual com grãos de pólen de flores do mesmo indivíduo, (4) xenogamia manual, 20 botões de flores pistiladas foram ensacados no dia anterior à antese e, após a abertura da flor, realizou-se a polinização manual com grãos de pólen de flores de indivíduos distantes pelo menos 200 metros da planta mãe, (5) Controle, 20 botões pistilados foram etiquetados e deixados acessíveis aos visitantes florais. Em todos os experimentos, utilizaram-se, no máximo, duas flores pistiladas por indivíduo e sacos de voal. A limitação de pólen foi mensurada a partir da comparação dos resultados obtidos nos tratamentos de autopolinização manual, polinização manual cruzada e controle. A razão pólen/óvulo foi estimada de acordo com Cruden (1977).

Visitantes florais - Foram feitas observações dos visitantes às flores, no período de março de 2004 a junho de 2007, em indivíduos focais no campo (10 indivíduos por espécie) com relação à frequência de visita às flores, comportamento, tempo de permanência e recurso coletado. No total foram feitas 132 horas de observações de campo para as três espécies sendo
96 horas distribuídas no período diurno (32 horas para cada espécie) e 36 horas no noturno (12 horas para cada espécie). As espécies de abelhas visitantes foram capturadas para identificação, sendo as mais comuns identificadas no campo, a partir de informações disponíveis em Pigozzo (2004). Material testemunho encontra-se depositado na coleção do Museu de Zoologia da Universidade Federal da Bahia. Os beija-flores foram identificados utilizando-se as pranchas do manual de campo proposto por Souza (2004) e a classificação seguiu as recomendações do Comitê Brasileiro de Registros Ornitológicos (Comitê Brasileiro de Registros Ornitológicos 2006).

Análise dos dados - Foi utilizada análise de variância não paramétrica Kruskal-Wallis (Siegel \& Castellan Junior 1988), ao nível de significância de 5\%, com teste de comparação múltipla de Dunn para analisar o efeito dos diferentes tratamentos dos sistemas reprodutivos na formação de frutos nas espécies estudadas, utilizando-se o programa GraphPad InStat@ 3.0 for Windows. Os gráficos de produção diária de néctar e de standing crop foram feitos no aplicativo R versão 2.13.0 (2011), pacote graphics version 2.13.0 e editados no aplicativo CorelDraw 12 versão 12.0.0.458 (2003).

\section{Resultados}

As três espécies de Jatropha estudadas compartilham atributos semelhantes. Todas são arbustos residentes na caatinga, decíduos, monóicos, com inflorescência do tipo dicásio. As flores são pentâmeras, do tipo disco (sensu Faegri \& Pij1 1979) e diclamídeas. Nas flores pistiladas o ovário é súpero, trilocular com um óvulo por lóculo. Há produção de néctar tanto nas flores pistiladas quantos nas estaminadas. Nas flores estaminadas as anteras são amarelas com deiscência rimosa. Os frutos são esquizocárpicos de deiscência explosiva, de coloração verde quando imaturos e marrom-acinzentados quando maduros. Detalhes da morfologia das inflorescências, flores e frutos de cada espécie estão organizados na tabela 1.

Biologia floral - As inflorescências de J. mollissima e J. mutabilis têm longevidade máxima de 32 e 25 dias, respectivamente. Em cada inflorescência, as primeiras flores a abrir são pistiladas, e somente após a senescência destas inicia-se a abertura das flores estaminadas. Após a abscisão das primeiras flores estaminadas, novas flores pistiladas podem abrir seguindo a mesma sequência descrita, de modo que não há sobreposição entre os dois tipos de flores na mesma inflorescência. Em novembro de 2006, observou-se em 12 indivíduos de J. mutabilis inflorescências contendo apenas flores estaminadas $(n=31)$. Nesse mesmo período, observaram-se nove flores estaminadas de J. mutabilis apresentando apenas rudimentos de estame e sem glândulas nectaríferas e 
Tabela 1. Comparação morfológica entre as inflorescências e flores, volume total, concentração média de néctar e total médio de açúcar por flor de Jatropha mollissima, J. mutabilis e J. ribifolia em Canudos, Bahia, nordeste do Brasil.

Table 1. Comparative morphology of inflorescences and flowers, nectar volume, concentration and total sugar per flower of Jatropha mollissima, J. mutabilis and J. ribifolia in Canudos, Bahia state, northeastern Brazil.

\begin{tabular}{|c|c|c|c|c|c|}
\hline Características & Sexo & $\mathrm{N}$ & J. mollissima & J. mutabilis & J. ribifolia \\
\hline \multicolumn{6}{|l|}{ Inflorescências E Flores } \\
\hline Comprimento da inflorescência (mm) & & 20 & $100-190$ & $40-50$ & $55-75$ \\
\hline Flores por inflorescência & $\begin{array}{l}\text { q } \\
\hat{0}\end{array}$ & $\begin{array}{l}30 \\
30\end{array}$ & $\begin{array}{c}2-4 \\
27-56\end{array}$ & $\begin{array}{c}1-2 \\
22-52\end{array}$ & $\begin{array}{c}2-3 \\
12-26\end{array}$ \\
\hline Diâmetro da corola (mm) & $\begin{array}{l}\text { o } \\
+ \\
\hat{0}\end{array}$ & $\begin{array}{l}20 \\
20\end{array}$ & $\begin{array}{l}25,5 \pm 1,73 \\
23,8 \pm 2,30\end{array}$ & $\begin{array}{l}21,6 \pm 2,65 \\
22,0 \pm 2,10\end{array}$ & $\begin{array}{l}7,8 \pm 1,10 \\
6,4 \pm 0,64\end{array}$ \\
\hline Frutos por inflorescência & q & 30 & $1-4$ & $1-2$ & $1-3$ \\
\hline \multicolumn{6}{|l|}{ Néctar } \\
\hline Volume total $(\mu \mathrm{L})$ & $\begin{array}{l}o \\
+ \\
\hat{0}\end{array}$ & $\begin{array}{l}10 \\
10\end{array}$ & $\begin{array}{l}107,0 \pm 36,8 \\
16,22 \pm 14,9\end{array}$ & $\begin{array}{c}133,17 \pm 16,8 \\
73,0 \pm 60,1\end{array}$ & $\begin{array}{c}1,05 \pm 0,55 \\
0,5 \pm 0,46\end{array}$ \\
\hline Concentração do néctar (\%) & $\begin{array}{l}\text { o } \\
+ \\
\hat{0}\end{array}$ & $\begin{array}{l}10 \\
10\end{array}$ & $\begin{array}{l}24,85 \pm 1,07 \\
23,08 \pm 1,12\end{array}$ & $\begin{array}{c}23,88 \pm 2,0 \\
21,81 \pm 2,48\end{array}$ & $\begin{array}{l}24,9 \pm 1,66 \\
23,5 \pm 1,58\end{array}$ \\
\hline Total de açucar médio por flor (mg) & $\begin{array}{l}\text { q } \\
\text { ô. }\end{array}$ & $\begin{array}{l}10 \\
10\end{array}$ & $\begin{array}{c}29,22 \\
4,03\end{array}$ & $\begin{array}{l}35,07 \\
17,51\end{array}$ & $\begin{array}{l}0,29 \\
0,13\end{array}$ \\
\hline
\end{tabular}

flores estaminadas normais permaneceram funcionais por até 36 horas. As inflorescências de J. ribifolia têm longevidade máxima de 18 dias. Abre-se, inicialmente, a flor pistilada mais central e, cerca de 24 a 48 horas depois, inicia-se a abertura das flores estaminadas. Com o amadurecimento da inflorescência, as demais flores pistiladas abrem-se concomitante com as flores estaminadas, ocorrendo sobreposição dos dois tipos de flores na mesma inflorescência.

Jatropha mollissima e J. mutabilis possuem abertura floral crepuscular e noturna e J. ribifolia, diurna. Em J. mollissima a movimentação das pétalas inicia-se às $17 \mathrm{~h} 30$ e às $20 \mathrm{~h} 00$ as flores estaminadas estão completamente abertas; já as pistiladas concluem a abertura às $21 \mathrm{~h} 30$. Em J. mutabilis, a partir das $16 \mathrm{~h} 30$ inicia-se a movimentação das pétalas, as quais às $19 \mathrm{~h} 00$, em ambos os tipos de flores, estão completamente abertas. O processo de movimentação das pétalas das flores de J. ribifolia é mais lento e pode se iniciar às $18 \mathrm{~h} 00$ do dia anterior à abertura da flor. O início de abertura, nos dois tipos florais e a exposição das estruturas reprodutivas ocorrem a partir das 4h30; a flor pistilada está completamente aberta às $7 \mathrm{~h} 00$ e a estaminada às $8 \mathrm{~h} 30$. Em todas as espécies, os estigmas ficam receptivos por toda a antese.

Em J. mollissima e J. mutabilis, as flores pistiladas têm duração de até 48 horas, permanecendo abertas durante todo o período. Entretanto, quando polinizadas, perdem as pétalas cerca de duas horas após receberem os grãos de pólen e, quando não ocorre polinização, até 36-48 horas o estigma e o ovário adquirem coloração amarronzada e murcham. As flores estaminadas têm longevidade máxima de 15 horas, quando ocorre a abscisão. Já em $J$. ribifolia, ambos os tipos de flores têm duração de até 48 horas, permanecendo abertas durante todo o período. Iniciada a senescência, ficam amarelas pálidas e caem. É comum a persistência das pétalas até a fase de amadurecimento dos frutos.

Nas três espécies, a deiscência das anteras ocorre no momento em que as estruturas reprodutivas ficam expostas a partir de um orifício formado pela separação das pétalas no ápice da flor e $100 \%$ dos grãos de pólen permanecem viáveis por toda a antese. Cada flor estaminada de $J$. mollissima produz, em média, $3516 \pm 268$ grãos de pólen, as de $J$. mutabilis, $2836 \pm 188$, e as de J. ribifolia, $1684 \pm 181$. Sendo assim, a razão pólen/óvulo foi, respectivamente, 1172,0; 945,3 e 561,3.

Em todas as espécies, o néctar começa a ser secretado cerca de meia hora após a abertura total da flor e a secreção permanece por toda a antese em $J$. ribifolia e até às $10 \mathrm{~h} 00$ (cerca de 17 horas após o início da antese) em $J$. mollissima e J. mutabilis. As flores 
pistiladas dessas duas espécies, que tem duração superior a 24 horas, interrompem a secreção de néctar às $10 \mathrm{~h} 00$ do primeiro dia de antese e retornam a produção às $19 \mathrm{~h} 00$, completando assim, dois períodos de secreção. Nas três espécies, $10 \%$ das flores estaminadas não produziam néctar. $\mathrm{O}$ volume de néctar secretado variou ao longo do dia e para J. mollissima e J. mutabilis o período de maior secreção ocorreu entre 01h30 e 04h00 (figura 1). Em J. mollissima e $J$. mutabilis houve disponibilidade de néctar (nectar standing crop) à noite, e ao amanhecer, já não havia mais néctar. Já em J. ribifolia, houve néctar durante todo o período (figura 2). Os dados de volume total e concentração média de néctar e total médio de açúcar por flor das três espécies são apresentados na tabela 1.

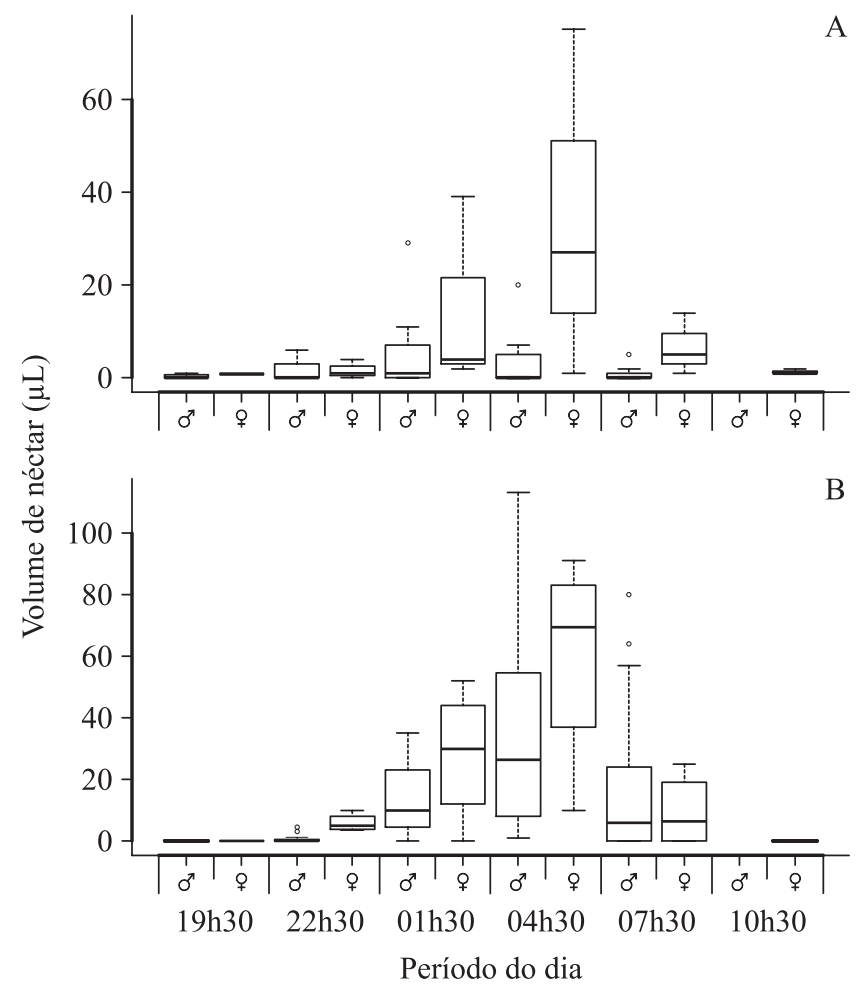

Figura 1. Box-plot da média da produção diária de néctar $(\mu \mathrm{L})$ em flores de Jatropha mollissima (A) e $J$. mutabilis (B) em Canudos, Bahia, nordeste do Brasil. As caixas compreendem $50 \%$ dos dados e a linha central marca a mediana. Limites internos e externos são definidos pela amplitude interquartis. Círculos indicam dados extremos.

Figure 1. Box-plot of mean daily nectar production in flowers of Jatropha mollissima (A) and J. mutabilis (B) in Canudos, Bahia state, northeastern of Brazil. The box comprises $50 \%$ of the data and the central line marks the median. Inner and outer fences are de ${ }^{\circledR n e d}$ by interquartile ranges. Circles indicate outliers.
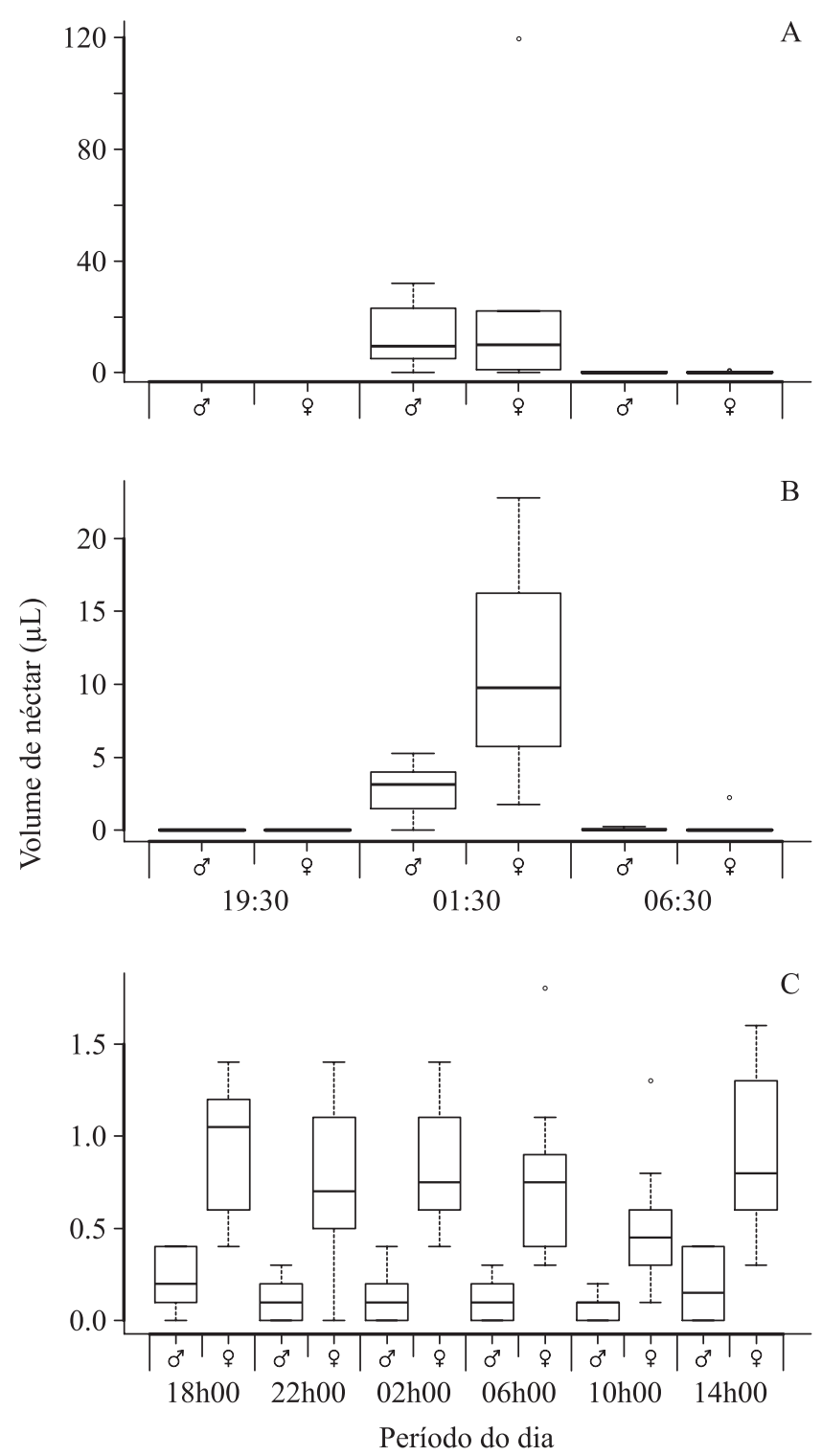

Figura 2. Box-plot da média do volume de néctar $(\mu \mathrm{L})$ disponível aos visitantes (standing crop) de $J$. mollissima (A), J. mutabilis (B) e J. ribifolia (C) em Canudos, Bahia, nordeste do Brasil. As caixas compreendem $50 \%$ dos dados e a linha central marca a mediana. Limites internos e externos são definidos pela amplitude interquartis. Círculos indicam dados extremos.

Figure 2. Box-plot of mean nectar standing crop volume $(\mu \mathrm{L})$ in J. mollissima (A), J. mutabilis (B) and J. ribifolia (C) in Canudos, Bahia state, northeastern of Brazil. The box comprises $50 \%$ of the data and the central line marks the median. Inner and outer fences are de ${ }^{\circledR}$ ned by interquartile ranges. Circles indicate outliers.

Sistemas reprodutivos - Os resultados dos experimentos dos sistemas reprodutivos das três espécies são apresentados na tabela 2. Apenas J. ribifolia produziu 
Tabela 2. Testes para verificação dos sistemas reprodutivos e formação de frutos em condições naturais (controle) de Jatropha mollisssima, J. mutabilis e J. ribifolia em Canudos, Bahia, nordeste do Brasil.

Table 2. Treatments for checking mating systems and fruit set under natural conditions (control) in Jatropha mollissima, J. mutabilis and J. ribifolia in Canudos, Bahia State, northeastern Brazil.

\begin{tabular}{|c|c|c|c|c|c|c|}
\hline \multirow{2}{*}{ Tratamento } & J. mollissima & \multirow{2}{*}{$\begin{array}{c}\text { Sucesso } \\
(\%)\end{array}$} & \multirow{2}{*}{$\begin{array}{c}\text { J. mutabilis } \\
\text { Flores/frutos }\end{array}$} & \multirow{2}{*}{$\begin{array}{c}\text { Sucesso } \\
(\%)\end{array}$} & \multirow{2}{*}{$\begin{array}{c}\text { J. ribifolia } \\
\text { Flores/frutos }\end{array}$} & \multirow{2}{*}{$\begin{array}{c}\text { Sucesso } \\
(\%)\end{array}$} \\
\hline & Flores/frutos & & & & & \\
\hline Apomixia & $20 / 1$ & $5 a$ & $20 / 0$ & $0 \mathrm{a}$ & $20 / 0$ & $0 \mathrm{a}$ \\
\hline Geitonogamia espontânea & $20 / 0$ & 0a & $20 / 0$ & 0a & $20 / 15$ & $75 b$ \\
\hline Geitonogamia manual & $20 / 16$ & $80 \mathrm{~b}$ & $20 / 15$ & $75 b$ & $20 / 18$ & $90 \mathrm{~b}$ \\
\hline Xenogamia manual & $20 / 17$ & $85 \mathrm{~b}$ & $20 / 17$ & $85 b$ & $20 / 18$ & $90 \mathrm{~b}$ \\
\hline Controle & $20 / 16$ & $80 \mathrm{~b}$ & $20 / 15$ & $75 b$ & $20 / 20$ & $100 \mathrm{~b}$ \\
\hline
\end{tabular}

Letras distintas representam valores significativos. Differente letters represent significative values.

frutos por geitonogamia espontânea. $\mathrm{O}$ fruto registrado no experimento de apomixia deve ser resultante de contaminação.

O teste de Kruskal-Wallis revelou que há diferença significativa entre os tratamentos para $J$. mollissima $(\mathrm{KW}=59,796)$, J. mutabilis $(\mathrm{KW}=59,058)$ e $J$. ribifolia $(\mathrm{KW}=63,660)$. Nota-se que $J$. molissima e $J$. mutabilis apresentaram o mesmo padrão de diferenças nos sucessos dos experimentos (tabela 2), onde a formação de frutos por geitonogamia manual, xenogamia manual e controle não diferiu entre si. O sucesso dos testes de geitonogamia manual, xenogamia manual e controle também não apresentou diferenças significativas para J. ribifolia, enquanto se observou que a formação de frutos por geitonogamia foi similar a estes tratamentos (tabela 2). Os frutos obtidos em todos os tratamentos, para as três espécies, possuíam três sementes e, em $J$. mollissima e $J$. mutabilis completaram a maturação entre 38 e 45 dias e em J. ribifolia, entre 26 e 32 dias.

Visitantes florais - As abelhas Apis mellifera, Trigona spinipes e Xylocopa grisescens visitaram as flores das três espécies, sendo A. mellifera a mais frequente, seguida de T. spinipes. O beija-flor Chlorostilbon lucidus foi o beija-flor mais frequente, visitando apenas as flores de $J$. mollissima e $J$. mutabilis, sendo esta última a mais visitada. As abelhas Eulaema nigrita, Xylocopa frontalis e Pseudoaugochlora pandora e o beija-flor Anopedia gounellei realizaram apenas visitas esporádicas (tabela 3). De modo geral, as visitas se concentraram no período das $06 \mathrm{~h} 00$ às $10 \mathrm{~h} 00$, tanto nas flores estaminadas quanto nas flores pistiladas, e poucos visitantes foram observados após o meio dia. Néctar foi o recurso mais procurado por todos os visitantes. Trigona spinipes, entretanto, foi mais frequentemente observada coletando pólen, tecido vegetal e seiva dos ramos. As visitas às flores de $J$. ribifolia foram raras durante todo o período.

Dentre as abelhas, apenas $A$. mellifera e $T$. spinipes visitaram várias flores (pistiladas e estaminadas) de um mesmo indivíduo de J. mollissima e J. mutabilis e permaneceram por mais tempo em cada flor. Ao visitarem as flores pistiladas, permaneciam pousadas nas pétalas coletando néctar e, após alguns segundos, se deslocavam ao redor do ovário contatando o estigma. Nas flores masculinas, T. spinipes coletou apenas pólen fixandose na antera para a retirada do mesmo com o auxílio das mandíbulas. Já $A$. mellifera, quando coletava néctar, introduzia o corpo no interior da flor por entre os estames e quando coletava pólen pousava diretamente sobre o ápice das anteras. Apis mellifera foi mais frequente e abundante nas flores de $J$. mutabilis.

As demais espécies visitaram apenas uma flor em cada indivíduo. Eulaema nigrita, $X$. grisescens e $X$. frontalis realizaram visitas rápidas e utilizaram as peças florais como local de pouso, contatando assim as estruturas reprodutivas. Pseudoaugochlora pandora visitou apenas as flores estaminadas onde ficavam em constante movimento e saiam com o corpo carregado de pólen.

Chlorostilbon lucidus e Anopedia gounellei são beija-flores pequenos e realizaram as visitas adejando a flor e introduzindo o bico diretamente na região das glândulas nectaríferas, contatando as estruturas florais em ambos os tipos de flores, raramente, visitando mais de duas flores por indivíduo. Observou-se que ambas as espécies percorreram distâncias maiores do que as abelhas ao saírem de uma planta para outra. O mesmo comportamento foi observado para as duas espécies de Xylocopa. Em 2006, foram observadas três visitas de morcego para tomada de néctar em flores pistiladas de J. mollissima, não sendo possível sua identificação. 
Tabela 3. Visitantes florais, número de visitas (n), duração da visita em segundos (dv) e recurso coletado (rc) em Jatropha mollissima, J. mutabilis e J. ribifolia, no período de março de 2004 a junho de 2007, em Canudos, Bahia, nordeste do Brasil ( $\mathrm{p}=$ pólen; $\mathrm{nc}=$ néctar $)$.

Table 3. Floral visitors, number of visits ( $n$ ), length of visits in seconds (dv) and resource collected (rc) in Jatropha mollissima, J. mutabilis and J. ribifolia from March 2004 to July 2007, in Canudos, Bahia State, northeastern Brazil (p = pollen; $\mathrm{nc}=$ nectar$)$.

\begin{tabular}{|c|c|c|c|c|c|c|c|c|c|}
\hline \multirow[b]{2}{*}{ Visitantes florais } & \multicolumn{3}{|c|}{ J. mollissima } & \multicolumn{3}{|c|}{ J. mutabilis } & \multicolumn{3}{|c|}{ J. ribifolia } \\
\hline & $n$ & $\begin{array}{l}\mathrm{dv} \\
(\mathrm{s})\end{array}$ & $\mathrm{rc}$ & $n$ & $\begin{array}{l}\mathrm{dv} \\
(\mathrm{s})\end{array}$ & $\mathrm{rc}$ & $n$ & $\begin{array}{l}\mathrm{dv} \\
(\mathrm{s})\end{array}$ & $\mathrm{rc}$ \\
\hline \multicolumn{10}{|l|}{ Abelhas (Hymenoptera, Apidae) } \\
\hline Apis mellifera (Linnaeus, 1758) & 100 & $3-15$ & $\mathrm{nc}, \mathrm{p}$ & 100 & $5-16$ & $\mathrm{nc}, \mathrm{p}$ & 10 & $4-9$ & $\mathrm{nc}$ \\
\hline Eulaema nigrita Lepeletier, 1841 & 2 & $2-6$ & nc & 5 & $2-6$ & nc & & & \\
\hline Pseudoaugoclora pandora (Smith, 1853) & 1 & & & 1 & $4-9$ & $\mathrm{nc}, \mathrm{p}$ & & & \\
\hline Trigona spinipes (Fabricius, 1793) & 50 & $5-80$ & $\mathrm{nc}, \mathrm{p}$ & 50 & $5-80$ & $\mathrm{nc}, \mathrm{p}$ & 20 & $5-80$ & $\mathrm{p}$ \\
\hline Xylocopa frontalis (Oliver, 1789) & & & & 9 & $2-3$ & $\mathrm{nc}, \mathrm{p}$ & & & \\
\hline Xylocopa grisescens Lepeletier, 1841 & 10 & $2-3$ & $\mathrm{nc}, \mathrm{p}$ & 20 & $2-5$ & $\mathrm{nc}, \mathrm{p}$ & 1 & $2-4$ & $\mathrm{nc}$ \\
\hline \multicolumn{10}{|l|}{ Beija-flores (Aves, Trochilidae) } \\
\hline Anopetia gounellei (Boucard, 1891) & & & & 4 & $2-3$ & nc & & & \\
\hline Chlorostilbon lucidus (Shaw, 1818) & 3 & $1-3$ & nc & 30 & $1-3$ & nc & & & \\
\hline \multicolumn{10}{|l|}{ Morcego (Chiroptera) } \\
\hline Indeterminado & 3 & $1-3$ & $\mathrm{nc}$ & & & & & & \\
\hline
\end{tabular}

\section{Discussão}

As três espécies apresentam flores com características que poderiam se encaixar em diferentes síndromes florais (sensu Faegri \& Pij1 1979, Proctor et al. 1996). De acordo com Ollerton et al. (2009) essa característica parece ser comum em diversas espécies de comunidades vegetais, tanto em regiões de clima tropical quanto em clima temperado. Para chegar a essa conclusão aqueles autores testaram experimentalmente a utilidade prática das síndromes florais e concluíram que raramente as características florais estão de acordo com as síndromes de polinização tradicionais. Tanto o volume quanto a concentração do néctar encontrados nos três representantes de Jatropha são compatíveis com os padrões encontrados para espécies melitófilas e ornitófilas (sensu Proctor et al. 1996), enquanto a cor vermelha das pétalas de $J$. mollissima e $J$. mutabilis é característica de flores ornitófilas e a cor esverdeada das flores de J. ribifolia, de flores miiófilas. Comportamento generalista na atração de visitantes também foi observado para J. mollissima e J. mutabilis (Santos et al. 2005) e J. curcas (Raju \& Ezradanam 2002, Bhattacharya et al. 2005).

O maior número de flores estaminadas em relação às pistiladas é uma estratégia que pode ser vantajosa para as plantas por aumentar a disponibilidade de pólen na população e, consequentemente, a chance de polinização cruzada. Essa característica é comum em espécies monóicas e dióicas e foi observado em outros estudos com espécies de Jatropha (p.e. Raju \& Ezradanam 2002, Añez et al. 2005, Bhattacharya et al. 2005, Santos et al. 2005). Além disso, essas flores recebem maior quantidade de visitas favorecendo a doação de maior quantidade de grãos de pólen (Stanton et al. 1986), compensando assim a sua menor longevidade (Silva et al. 2006).

Em $J$. mollissima e $J$. mutabilis a abertura das flores pistiladas antes das estaminadas em uma mesma inflorescência, fenômeno chamado de protoginia interfloral (Lloyd \& Webb 1986), aumenta as chances de alogamia. Entretanto, como ocorre sincronia na antese de flores pistiladas e estaminadas, em inflorescências diferentes do mesmo indivíduo, pode ocorrer geitonogamia (Silva et al. 2006). Na área deste estudo, a geitonogamia parece ser potencializada devido ao comportamento de visita a diversas flores do mesmo indivíduo realizada por $A$. mellifera, principal polinizador em ambas as espécies (Neves \& Viana 2011). Em $J$. ribifolia, apesar das flores não serem bissexuadas e de existir protoginia na inflorescência, as flores pistiladas de uma mesma inflorescência abrem e permanecem viáveis em sobreposição temporal com as flores estaminadas, 
favorecendo a geitonogamia. Uma característica que também parece favorecer a polinização dessa espécie, que atrai poucos visitantes, é a maior longevidade das flores pistiladas.

Jatropha mollissima e J. mutabilis apresentaram variação nos eventos de desenvolvimento da inflorescência e da flor em relação aos observados por Santos et al. (2005). Dentre eles, destaca-se o horário de início da abertura das flores que, em Canudos, foi vespertina com as flores estaminadas e pistiladas permanecendo abertas durante a noite, enquanto em Pernambuco, foi matutina iniciando-se às $04 \mathrm{~h} 00$. Esse resultado sugere que os eventos de desenvolvimento floral em Jatropha são flexíveis e influenciados por variações espaciais e ambientais.

Em plantas com flores unissexuais, uma modificação de sexo do indivíduo pode acontecer rapidamente através de estímulo ambiental, do tamanho ou idade do indivíduo (Richards 1997). Um exemplo disso é a dioicia funcional, ou seja, alternância de função pistilada e estaminada em um mesmo indivíduo, tornando-o temporariamente unissexual. Esse fenômeno, que ocorre em pelo menos 37 famílias de angiospermas, está fortemente associado à monoicia, à protoginia e à autocompatibilidade, e tem como principal vantagem evolutiva minimizar a geitonogamia e favorecer a xenogamia (Cruden 1988). Dioicia funcional foi observada em J. mutabilis, cujas inflorescências apresentavam, exclusivamente flores estaminadas, tornando os indivíduos funcionalmente masculinos. Não foram encontrados registros para outras espécies de Jatropha, mas Raju \& Ezradanam (2002) descreveram para $J$. curcas a ocorrência de indivíduos com algumas inflorescências contendo flores estaminadas na posição de flores pistiladas, evidenciando a possibilidade de sua ocorrência. Santos et al. (2005) registraram a ocorrência de flores em estágio intermediário de sexualidade, com vestígio do sexo oposto em $J$. mollissima e $J$. mutabilis, mas não mencionam fenômenos de dioicia funcional.

Em J. ribifolia, a produção de néctar até a senescência das flores pode ser considerada mais uma estratégia desta espécie para a atração de potenciais polinizadores. Entretanto, isso não pode ser comprovado devido ao baixo número de visitantes observados durante o período amostral. Para J. mollissima e J. mutabilis, o horário de início da produção de néctar foi semelhante àquele observado por Santos et al. (2005). Entretanto, aquelas autoras relataram que não houve produção de néctar à noite, nem mesmo nas flores pistiladas que duram mais de dois dias, diferente do aqui relatado, uma vez que a maior produção, nas três espécies, ocorreu durante a madrugada. A diminuição do volume em $J$. mollissima e $J$. mutabilis ao longo do dia deve estar relacionado ao aumento da temperatura e diminuição da umidade relativa, conforme sugerem Faegri \& Pijl (1979). Outra possibilidade é que tenha havido reabsorção do néctar, recurso que permite às plantas economizarem metabólitos não consumidos pelos polinizadores (Luyt \& Johnson 2002).

Maior quantidade de açúcar disponibilizado no néctar produzido pelas flores pistiladas deve ampliar sua atratividade aos visitantes e com isso aumentar as chances de polinização, já que são menos abundantes em todos os indivíduos. Por outro lado, as flores estaminadas, mais abundantes, disponibilizam menor quantidade de açúcar, o que, de acordo com Bhattacharya et al. (2005), representa um ganho evolutivo estabelecido graças às forças seletivas operando no sistema planta-polinizador. A maior frequência e abundância de A. mellifera nas flores de J. mutabilis pode ser explicada pela maior quantidade de açúcar disponibilizada por esta espécie vegetal, tanto nas flores pistiladas, quanto nas estaminadas.

De acordo com a classificação de Cruden (1977) para a relação pólen/óvulo, as três espécies apresentam xenogamia facultativa. Jatropha ribifolia, que apresentou a menor relação pólen/óvulo e foi observada produzindo frutos sem a participação de vetores bióticos, parece ser a espécie mais flexível. Jatropha mollissima e $J$. mutabilis são autocompatíveis, mas, por necessitarem, obrigatoriamente, de vetores de pólen, a xenogamia deve representar o único modo de reprodução. Entretanto, essa constatação não afasta a possibilidade de endogamia, já que $A$. mellifera, visitante mais frequente, coleta em várias flores de um mesmo indivíduo e, quando muda de planta, tende a ir para o indivíduo mais próximo (Neves \& Viana 2011). Sabe-se que espécies monóicas autocompatíveis estão propensas a altas taxas de autofecundação, que podem resultar em depressão por endocruzamento (Cruden 1988b). Esse comportamento foi observado em $J$. ribifolia que, durante o período amostrado, recebeu raras visitas às flores e apresentou altas taxas de autopolinização espontânea, sugerindo ser esse o principal meio de reprodução desse táxon no período amostrado.

A alta frequência e abundância das espécies eussociais $A$. mellifera e $T$. spinipes nas flores foram observadas em diversos estudos sobre visitantes florais e biologia da polinização em vários biomas brasileiros (e.g. Wilms et al. 1996, Neves \& Viana 2002, Klein et al. 2003, Minussi \& Alves-dos-Santos 2007). Em Canudos, esse resultado pode ter sido ainda mais acentuado pela habilidade dessas abelhas em nidificarem nas frestas dos 
paredões de arenito dos canyons e, no caso de $A$. mellifera, pela dificuldade dos meleiros em predar suas colônias devido à dificuldade de acesso. Em estudo realizado na área por Pigozzo \& Viana (2010) em relação às redes de interações estabelecidas entre as espécies de abelhas e plantas, $11,5 \%$ e $10,1 \%$ das interações aconteceram entre a flora local e as abelhas $A$. mellifera e $T$. spinipes, respectivamente. Esse resultado evidencia a importância relativa dessas abelhas no sistema planta-polinizador dessa região.

O baixo número de indivíduos de abelhas nativas e de beija-flores observado em $J$. mollissima e $J$. mutabilis, em todos os períodos de observação, pode ter sido resultante do monopólio de $A$. mellifera nas flores, impedindo a aproximação dos demais visitantes, já que essas abelhas iniciam as visitas a partir das $04 \mathrm{~h} 30$, com grande número de indivíduos por planta. Às 06h00, quando se inicia a atividade dos demais visitantes, elas já coletaram a quase totalidade dos grãos de pólen e do néctar disponíveis. A exaustão de recursos florais por A. mellifera, deslocando visitantes nativos, também foi observada por Paton (1993) e Celebrezze \& Paton (2004) na Austrália. Na área do presente estudo, essa evidência preocupa porque se $A$. mellifera estiver deslocando os visitantes nativos devido à exaustão dos recursos florais, espécies vegetais que dependam exclusivamente de polinizadores nativos terão seu sucesso reprodutivo influenciado negativamente.

$\mathrm{O}$ registro de visitas de morcegos em flores de Jatropha é inédito. O fato desses mamíferos terem sido observados apenas em flores pistiladas deve estar relacionado ao tipo floral, o qual expõe completamente o néctar acumulado, e à grande quantidade produzida que, muitas vezes, escorre entre as pétalas. A tomada em flores estaminadas é dificultada pelo menor diâmetro da flor e preenchimento do espaço interno pelos estames. Como não foram registradas visitas às flores estaminadas, não é possível classificar os morcegos como polinizadores efetivos de J. mollissima. Embora sem apresentar atributos florais característicos de quiropterofilia (com exceção do horário de abertura das flores), é possível que, em períodos de escassez de recursos, a exploração de néctar das flores de $J$. mollisima por morcegos se intensifique e esses mamíferos possam vir a atuar como polinizadores ocasionais e elementos de pressão seletiva que resultem em mudança de síndrome floral.

As observações sobre o comportamento dos visitantes sugerem que em Canudos as abelhas Apis mellifera, Xylocopa frontalis e X. grisescens e os beija-flores Anopetia gounellei e Chlorostilbon lucidus seriam os polinizadores efetivos de $J$. mollissima e $J$. mutabilis. Santos et al. (2005) sugerem que essas espécies podem ser polinizadas, também, por vespas e borboletas, mas indivíduos desses grupos não foram amostrados em Canudos. A pequena abundância de A. mellifera visitando flores de $J$. ribifolia pode estar relacionada à menor quantidade de indivíduos, e consequentemente de flores, disponíveis dessa espécie vegetal em relação às outras duas espécies de Jatropha. É conhecido que $A$. mellifera forrageia, preferencialmente, em plantas que disponibilizam grande quantidade de flores (Wilms et al. 1996), explorando, portanto, outras fontes de recursos mais produtivas. Distintas espécies de abelhas eussociais também parecem ser visitantes potenciais de J. ribifolia na área, apesar de não terem sido amostradas nas flores. Em estudo a respeito do potencial apícola de três espécies de Mimosa L., realizado na mesma área do presente trabalho, Lima (2007) registrou a presença de pólen de J. ribifolia armazenado em colônias artificiais de Plebeia sp., indicando que táxons desse gênero forrageiam em flores dessa espécie.

Os dados obtidos neste estudo permitem concluir que a monoicia favorece a xenogamia nestas espécies, mas o sucesso desse sistema de reprodução depende do desenvolvimento assincrônico das inflorescências e do comportamento dos polinizadores.

Agradecimentos - À Fundação Biodiversitas pela autorização concedida para o desenvolvimento dos trabalhos de campo na Estação Biológica de Canudos; à Fundação de Amparo à Pesquisa do Estado da Bahia Fapesb (Termo de Outorga TO 261/03 Etapa II) pelo apoio financeiro às atividades de campo; ao CNPq pela bolsa de produtividade em pesquisa a Blandina F. Viana e Isabel C. Machado; aos membros do Labea/IBUFBA pelo auxílio nas coletas de campo.

\section{Referências bibliográficas}

AÑEZ, L.M.M., COELHO, M.F.B., ALBUQUERQUE, M.C.F. \& DOMBROSKI, J.L.D. 2005. Caracterização morfológica dos frutos, sementes e do desenvolvimento das plântulas de Jatropha elliptica Müll. Arg. (Euphorbiaceae). Revista Brasileira de Botânica 28: 563-568.

BHATTACHARYA, A., DATTA, K. \& DATTA, S.K. 2005. Floral biology, floral resource constraints and pollination limitation in Jatropha curcas L. Pakistan Journal of Biological Sciences 8:456-460.

BULLOCK, S.H. 1985. Breeding systems in the flora of a tropical deciduous forest in Mexico. Biotropica 17: 287-301. 
CELEBREZZE, T. \& PATON, D.C. 2004. Do introduced honeybees (Apis mellifera, Hymenoptera) provide full pollination service to bird-adapted Australian plants with small flowers? An experimental study of Brachyloma ericoides (Epacridaceae). Austral Ecology 29:129-136.

COMITÊ BRASILEIRO DE REGISTROS ORNITOLÓGICOS. 2006. Listas das aves do Brasil. Versão 2006. http://www.ib.usp.br/cbro (acesso em 29/04/2007).

CRUDEN, R.W. 1977. Pollen-ovule ratios: a conservative indicator of breeding systems in flowering plants. Evolution 31:32-46.

CRUDEN, R.W. 1988. Temporal dioecism: systematic breadth, associated traits, and temporal patterns. Botanical Gazette 149:1-15.

DAFNI, A., KEVAN, P.G. \& HUSBAND, B.C. 2005. Practical pollination ecology. Environquest Ltd., Ontario.

DEHGAN, B. \& WEBSTER, G.L. 1979. Morphology and infrageneric relationships of the genus Jatropha (Euphorbiaceae). University of California Press, Berkeley.

EPILA-OTARA, J.S.S. 1993. Foraging behavior of honeybees (Hymenoptera: Apidae) on Jatropha integerrima (Euphorbiaceae) in Upolu, Western Samoa. Micronesica 26:83-94.

FAEGRI, K. \& VAN DER PIJL, L. 1979. The principles of pollination ecology. Pergamon Press, Oxford.

FRANKIE, G.W., HARBER, W.A., OPLER, P.A. \& BAWA, K.S. 1983. Characteristics and organization of the large bee pollination systems in the Costa Rican dry forest. In Handbook of experimental pollination biology (C.E. Jones \& R.J. Little, eds.). Van Nostrand Reinhold Company Inc., New York, p.411-448.

GALETTO, L. \& BERNADELlO, G. 2005. Nectar. In Practical pollination ecology (A. Dafni, P.G. Kevan \& B. C. Husband, eds.). Environquest Ltd., Ontario, p.261-313.

GIUlIETTI, A.M., CONCEIÇÃO, A. \& QUEIROZ, L.P. 2006. Diversidade e caracterização das fanerógamas do semi-árido brasileiro. Associação Plantas do Nordeste, Recife, v.1.

KEARNS, C.A. \& INOUYE, D.W. 1993. Techniques for pollination biologists. University Press of Colorado, Colorado.

KLEIN, A.M., STEFFAN-DEWENTER, I. \& TSCHARNTAKE, T. 2003. Pollination of Coffea canephora in relation to local and regional agroforestry management. Journal of Applied Ecology 40:837-845.

LIMA, L.C.L. 2007. Espécies de Mimosa L. (Leguminosae) do semi-árido nordestino: palinologia, fenologia, biologia floral e potencial apícola. Tese de doutorado, Universidade Estadual de Feira de Santana, Feira de Santana.

LLOYD, D.G. \& WEBB, C.J. 1986. The avoidance of interference between the presentation of pollen and stigmas in angiosperm. I. Dichogamy. New Zealand Journal of Botany 24:135-162.
LUYT, R. \& JOHNSON, S.D. 2002. Postpollination nectar reabsorption and its implications for fruit quality in an epiphytic orchid. Biotropica 34:442-446.

MINISTÉRIO DO MEIO AMBIENTE. 2002. Avaliação e ações prioritárias para a conservação da biodiversidade da Caatinga. MMA, Brasília.

MINUSSI, L.C. \& ALVES-DOS-SANTOS, I. 2007. Abelhas nativas versus Apis mellifera Linnaeus, espécie exótica (Hymenoptera: Apidae). Bioscience Journal 23: 58-62.

MUCHALA, N. 2003. Exploting the boundary between pollination syndromes: bats and hummingbirds as pollinators of Burmeistera cyclostigmata and $B$. tenuiflora (Campanulaceae). Oecologia 134:373-380.

NEVES, E.L. \& VIANA, B.F. 2002. As abelhas eussociais (Hymenoptera, Apidae) visitantes florais em um ecossistema de dunas continentais no médio Rio São Francisco, Bahia, Brasil. Revista Brasileira de Entomologia 46:571-578.

NEVES, E.L. \& VIANA, B.F. 2011. Pollination efficiency of Apis mellifera Linnaeus, 1758 (Hymenoptera, Apidae) on the monoecious plants Jatropha mollissima (Pohl) Baill. and Jatropha mutabilis (Pohl) Baill. (Euphorbiaceae) in a semi-arid Caatinga area, northeastern Brazil. Brazilian Journal of Biology 71: 107-113.

NEVES, E.L., FUNCH, L.S. \& VIANA, B.F. 2010. Comportamento fenológico de três espécies de Jatropha (Euphorbiaceae) da Caatinga, semi-árido do Brasil. Revista Brasileira de Botânica 33:155-166.

OLLERTON, J., AL R., WASER, N.M., PRICE, M.V., WATTS, S., CRANMER, L., HINGSTON, A. PETER, C.I. \& ROTENBERRY, J. 2009. A global test of the pollination syndrome hypothesis. Annals of Botany 103:1471-1480.

ORMOND, W.T., PINHEIRO, M.C.B. \& CASTELLS, A.R.C. 1984. Contribuição ao estudo da reprodução e biologia floral de Jatropha gossypifolia L. (Euphorbiaceae). Revista Brasileira de Biologia 44:159-167.

PATON, D.C. 1993. Honeybees Apis mellifera in the Australian environment. Does Apis mellifera disrupt or benefit native biota? Bioscience 43:95-103.

PIGOZZO, C.M. 2004. Organização das comunidades locais de Apoidea em uma área de Caatinga, Canudos - BA. Dissertação de mestrado, Universidade Federal da Bahia, Salvador.

PIGOZZO, C.M. \& VIANA, B.F. 2010. Estrutura da rede de interações entre flores e abelhas em ambiente de Caatinga. Oecologia Australis 14:10-114.

PRIMACK, R.B. 1985. Longevity of individual flowers. Annual Review of Ecology and Systematics 16: $15-37$.

PROCTOR, M., YEO, P. \& LACK, A. 1996. The natural history of pollination. Harper Collins Publishers, London. 
RAJU, A.J.S. \& EZRADANAM, V. 2002. Pollination ecology and fruiting behavior in a monoecious species, Jatropha curcas L. (Euphorbiaceae). Current Science 83:1395-1398.

REDDI, E.U.B. \& REDDI, C.S. 1983. Pollination ecology of Jatropha gossypifolia (Euphorbiaceae). Proceedings of the Indian Academy of Sciences 92:215-231.

RICHARDS, A.J. 1997. Plant breeding systems, 2 $2^{\mathrm{a}}$ ed. Chapman \& Hall, London.

ROUBIK, D.W. 1995. Pollination of cultivated plants in the tropics. FAO Agricultural Services Bulletin, v.118, Food and Agriculture Organization of the United Nations, Rome.

SANTOS, M.J., MACHADO, I.C. \& LOPES, A.V. 2005. Biologia reprodutiva de duas espécies de Jatropha L. (Euphorbiaceae) em Caatinga, Nordeste do Brasil. Revista Brasileira de Botânica 28:361-373.

SIEGEL, S. \& CASTELLAN JÚNIOR, N. 1988. Nonparametric statistics for the behavior sciences. McGraw-Hill, New York.
SILVA, L.M.M., AGUIAR, I.B., VIÉGAS, R.A. \& MENDONÇA, I.F.C. 2006. Biologia reprodutiva de Cnidosculus juercifolius Pax \& K. Hoffm (Euphorbiaceae). Revista de Biologia e Ciências da Terra 6:25-34.

SOUZA, D. 2004. Todas as aves do Brasil. Dall, Feira de Santana.

STANTON, M.L., SNOW, A.A. \& HANDEL, S.N. 1986. Floral evolution: attractiveness to pollinators increases male fitness. Science 232:1625-1627.

VELLOSO, A.L., SAMPAIO, E.V.S.B. \& PAREYN, F.G.C. (eds.). 2002. Ecorregiões: propostas para o Bioma Caatinga. TNC-Brasil, Associação Plantas do Nordeste, Recife.

WILMS, W., IMPERATRIZ-FONSECA, V.L. \& ENGELS, W. 1996. Resource partitioning between highly eusocial bees and possible impact of introduced Africanized honey bees on native Stingless bees in Brazilian Atlantic Rainforest. Studies on Neotropical Fauna \& Environment 31:137-151. 